\title{
Anatomical Single-bundle Anterior Cruciate Ligament Reconstruction Using a Freehand Transtibial Technique
}

\author{
Kyung-Wook Nha, $\mathrm{MD}^{1}$, Jae-Hwi Han, $\mathrm{MD}^{1}$, Jae-Ho Kwon, $\mathrm{MD}^{2}$, Kyung-Woon Kang, $\mathrm{MD}^{1}$, \\ Hyung-Joon Park, $\mathrm{MD}^{1}$, and Jae-Gwang Song, $\mathrm{MD}^{1}$ \\ ${ }^{1}$ Department of Orthopaedic Surgery, Inje University Ilsan Paik Hospital, Goyang; ${ }^{2}$ Department of Orthopaedic Surgery, Barunsesang Hospital, Seongnam, Korea
}

\begin{abstract}
Introduction: In anatomical single-bundle (SB) anterior cruciate ligament (ACL) reconstruction, the traditional transtibial approach can limit anatomical placement of the femoral tunnel.

Surgical Technique: We present a novel three-point freehand technique that allows for anatomic SB ACL reconstruction with the transtibial technique.

Materials and Methods: Between January 2012 and December 2012, 55 ACL reconstructions were performed using the three-point freehand technique. All the patients were followed for a minimum of 12 months post-operatively. Clinical evaluation was done using the Lysholm score and International Knee Documentation Committee (IKDC) grade. All patients were analyzed by 3-dimensional computed tomography (3D CT) at 1 week after surgery.

Results: The mean Lysholm score improved from $68.2 \pm 12.7$ points preoperatively to $89.2 \pm 8.2$ points at final follow-up. At final follow-up, the IKDC grade was normal in 42 patients and nearly normal in 13 patients. None of the patients had a positive pivot shift test, anterior drawer test and Lachman test at final follow-up. The anatomical position of the femoral tunnel was confirmed on 3D CT scans.

Conclusions: The three-point freehand technique for SB transtibial ACL reconstruction is a simple, anatomic technique showing good clinical results.
\end{abstract}

Keywords: Anterior cruciate ligament, Reconstruction, Single-bundle, Transtibial technique

\section{Introduction}

Various techniques for anterior cruciate ligament (ACL) reconstruction have been recently introduced. There are approaches to femoral tunnel placement in arthroscopic ACL reconstruction: the tibial tunnel-dependent approach (transtibial technique) and the tibial tunnel-independent approach (anteromedial [AM] and outside-in techniques). Femoral tunnel placement using the trans-

Received July 14, 2014; Revised (1st) October 12, 2014;

(2nd) December 9, 2014; Accepted January 20, 2015

Correspondence to: Jae-Gwang Song, MD

Department of Orthopaedic Surgery, Inje University Ilsan Paik Hospital, 170 Juhwa-ro, Ilsanseo-gu, Goyang 411-706, Korea

Tel: +82-31-910-7312, Fax: +82-31-910-7319

E-mail: sjgwang@naver.com

This is an Open Access article distributed under the terms of the Creative Commons Attribution Non-Commercial License (http://creativecommons.org/licenses/by-nc/4.0/) which permits unrestricted non-commercial use, distribution, and reproduction in any medium, provided the original work is properly cited. tibial technique is a common and relatively easy procedure in single-bundle (SB) ACL reconstruction. However, recent reports and emphasis on anatomic tunnel placement have generated the need to reconsider the application of the transtibial approach. Some authors reported that the femoral tunnels created using the transtibial approach are non-anatomic ${ }^{1-3)}$. So, there have been some efforts to modify the transtibial technique by positioning the starting point of the tibial tunnel more medial and proximal for a more oblique trajectory of the femoral tunnel ${ }^{2}$. However, this has led to other problems such as a shorter tibial tunnel and widening of the intra-articular aperture of the tibial tunnel ${ }^{3.4)}$. Therefore, a transition to creation of a femoral tunnel independent of the tibial tunnel is recommended to achieve anatomic femoral tunnel placement ${ }^{5)}$. Techniques for creating anatomical femoral footprint in SB reconstruction have been reported, such as the trans-AM portal technique ${ }^{6,7)}$ and the outside-in technique $^{8)}$. However, disadvantages such as insufficient femoral tunnel length, posterior wall breakage and a bent graft would limit 


\section{Nha et al. Anatomic ACL Reconstruction Using Freehand Technique}

the use of trans-AM portal technique ${ }^{9)}$. Similarly, acute femoral tunnel angle, inadequate femoral fixation and additional lateral skin incisions upon surgery are major hindrances for the use of the outside-in technique ${ }^{10)}$. To overcome the problems of the above-described modified transtibial technique and tibial tunnel independent technique, we devised a three-point freehand transtibial technique. We designed the three-point freehand trantibial technique for the purpose of anatomic femoral tunnel placement and evaluated the tunnel position with 3-dimensional computed tomography (3D CT) to determine clinical significance.

\section{Surgical Technique}

A standard anterolateral (AL) portal is placed for diagnostic arthroscopy and an AM portal as a working portal. Following an arthroscopic examination of the knee joint, the autohamstring tendons (both semitendinosus and gracilis tendons) are harvested using a closed-end graft harvester through an oblique skin incision along the distal insertion of the tendons on the proximal tibia and prepared as a 4-strand double loop graft. Femoral notchplasty is performed when the presence of notch osteophytes and/or impingement of the graft against the notch are expected. For tibial tunnel placement, a tibial drill guide (ConMed Linvatec, Largo, FL, USA) is set at $45^{\circ}$ from the starting point at the midway between the posterior cortex of the proximal tibia and the medial margin of the tibial tuberosity that is just anterior to the superficial medial collateral ligament. Under direct arthroscopic visualization, the center of the tibial footprint is marked with a $45^{\circ}$ microfracture instrument (ConMed Linvatec) and the tip of the tibial drill guide is positioned at the center of the tibial footprint. The guide pin is inserted through the tibial drill guide and the tibial tunnel is created using a cannulated reamer with the same diameter of the graft. After reaming of the tibial tunnel, the femoral tunnel is prepared by the three-point freehand technique. First, the $7 \mathrm{~mm}$ femoral offset aimer (ConMed Linvatec) is introduced through the tibial tunnel for preparation of the reference point. The femoral offset aimer is placed at around the 10:30 clock position (right knee)/1:30 clock position (left knee) of the over-the-top area of the lateral intercondylar notch and rotated laterally ${ }^{11)}$. The guide pin is inserted through the offset aimer to mark the reference point which is more proximal ${ }^{12)}$ than the center of the femoral footprint due to offset hook (Figs. 1A and 2A). After creating the reference point, the guide pin is removed. Second, the center of the femoral footprint is marked with a femoral offset aimer that is introduced through the AM portal with the knee positioned at $90^{\circ}$ of flexion. Under direct arthroscopic visualization, the center of the femoral footprint is located more posterior and distal ${ }^{12)}$ to the reference point created by the femoral offset aimer (Figs. 1B and 2B). After marking the center of femoral footprint, the guide pin is withdrawn from the joint. Finally, the cannulated femoral guide pin is introduced through the tibial tunnel by the freehand technique without a femoral offset aimer and positioned at the previously marked center of the footprint (Figs. 1C and 2C). The guide pin is drilled through the femur and passed out of the lateral cortex of the femur. The reamer is advanced through the tibial tunnel and the femoral tunnel is created with the knee positioned at $70^{\circ}$ flexion. The 4-strand double looped autohamstring tendon graft is pulled through the tibial tunnel to the femoral tunnel. The graft is secured on the femoral side by use of the RigidFix system (DePuy Mitek Inc., Raynham, MA, USA). At the tibial tunnel, the graft is fixed with the IntraFix biodegradable screw (DePuy Mitek Inc.) device with the knee in $10^{\circ}$ flexion and supplemented by a screw and a spike washer. After fixation of the graft, the arthroscope is reintroduced to confirm tension and to rule out impingement of the graft.
A

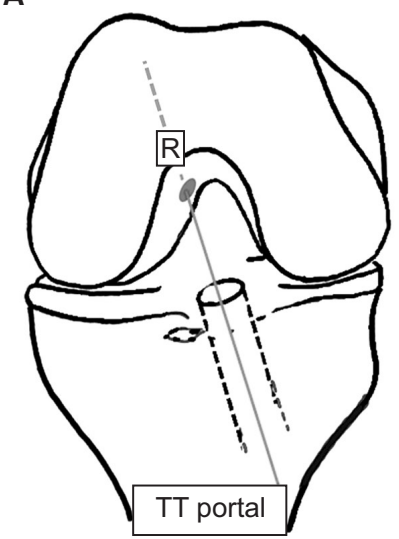

B

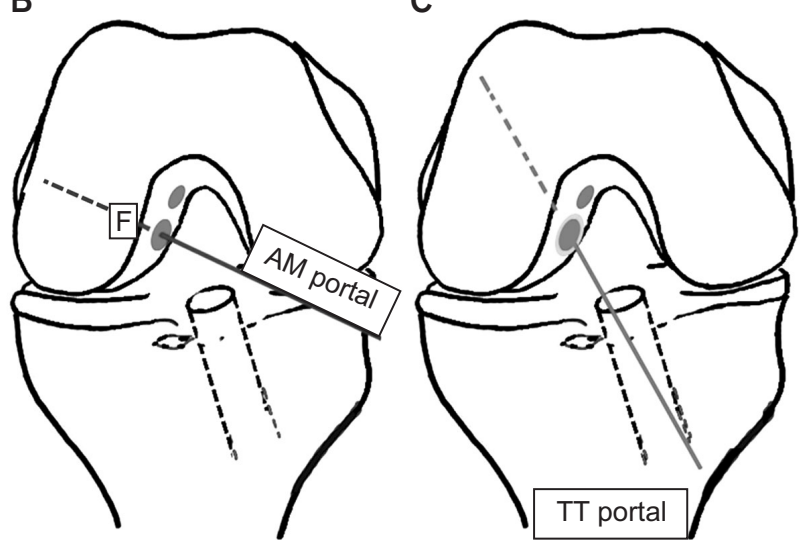

Fig. 1. (A) The reference point $R$ was marked according to the modified transtibial technique with a femoral offset aimer. (B) The center of femoral footprint F was marked through the anteromedial portal. (C) The cannulated femoral guide pin passed through the tibial tunnel accoding to the freehand technique is positioned at the center of the footprint. R: reference point, TT: transtibial, F: footprint, AM: anteromedial. 

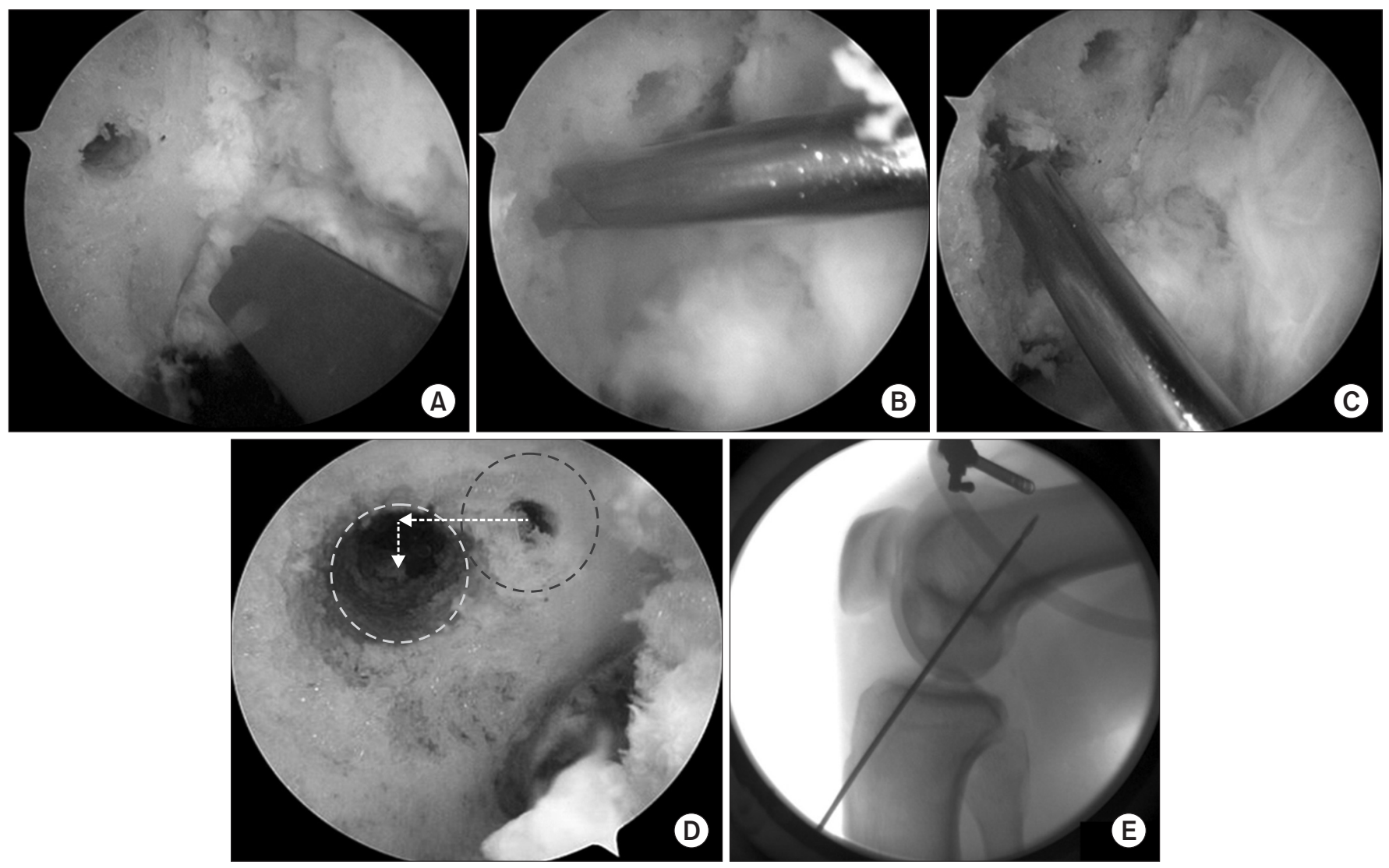

Fig. 2. Arthroscopic images of anterior cruciate ligament (ACL) reconstruction using the three-point freehand transtibial technique: (A) A reference point was marked by a femoral offset aimer; (B) A guide pin was introduced through the anteromedial portal; (C) The guide pin was positioned at the center of femoral footprint through tibia tunnel; and (D) after drilling, the femoral tunnel was placed to be more posterior and distal to the reference point. (E) Lateral fluoroscopic view showing more distal position of the femoral tunnel than that in ACL reconstruction using a modified transtibial technique.

Table 1. Patient Demographics

\begin{tabular}{lc}
\hline \multicolumn{1}{c}{ Variable } & Data (range) \\
\hline No. of patients & 55 \\
Age (yr) & $35.6(19-46)$ \\
Gender (M:F) & $36: 19$ \\
Combined Injury & \\
Meniscal injury & 35 \\
MCL injury & 13 \\
PLRI & 2 \\
Operation time (min) & $58(48-74)$ \\
Follow-up (mo) & $14(12-23)$ \\
\hline
\end{tabular}

MCL: medial collateral ligament, PLRI: posterolateral rotatory instability.

\section{Materials and Methods}

Between January 2012 and December 2012, 55 ACL reconstructions were performed using the three-point freehand technique.

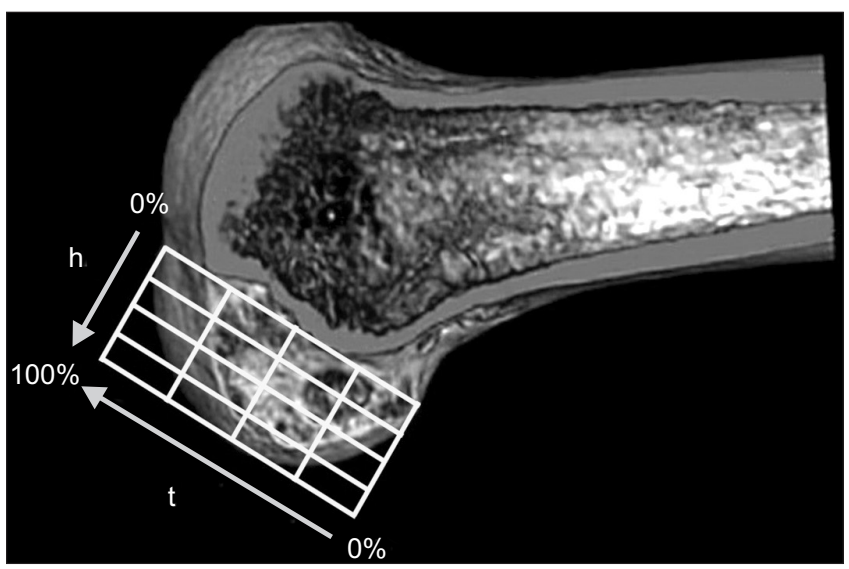

Fig. 3. Femoral tunnel position by the quadrant method in a 3-dimensional computed tomography model. t: line parallel to the Blumensaat's line, h: line perpendicular to the Blumensaat's line.

All the patients were followed for a minimum of 12 months postoperatively. The patients' average age was 35.6 years (range, 19 to 
46 years). The mean follow-up period was 14 months (range, 12 to 23 months) (Table 1). Evaluation of clinical results was done using the Lysholm score and objective International Knee Documentation Committee (IKDC) grade. All patients underwent 3D CT scanning (Aquilion One; Toshiba Medical Systems Cor., Otawara, Japan) at 1 week after surgery. The created 3D CT images were aligned with the femoral tunnels to perform analysis according to the quadrant method suggested by Bernard et al. ${ }^{13)}$ using PACS View (PACS; Marotech Inc., Seoul, Korea) (Fig. 3).

\section{Results}

\section{Clinical Assessment}

All the patients showed good or excellent clinical outcomes in the Lysholm score with a median score of 89.2 at final follow-up. The IKDC grade at final follow-up was normal in 42 patients and nearly normal in 13 patients. None of the patients had a positive pivot shift test, anterior drawer test and Lachman test at final follow-up. The mean side-to-side difference in anterior translation on the Telos stress radiography was $7.1 \pm 1.7 \mathrm{~mm}$ preoperatively

Table 2. Clincal Results

\begin{tabular}{lcc}
\hline \multicolumn{1}{c}{ Characteristics } & Preoperative & Postoperative \\
\hline Modified Lysholm Score & $68.2 \pm 12.7$ & $89.2 \pm 8.2$ \\
IKDC grade & & \\
Normal & 0 & 42 \\
Nearly normal & 0 & 13 \\
Abnormal & 22 & 0 \\
Severely abnormal & 23 & 0 \\
Telos stress X-ray (side-to-side difference) $(\mathrm{mm})$ & $7.1 \pm 1.7$ & $1.7 \pm 1.2$ \\
\hline
\end{tabular}

Values are presented as mean \pm standard deviation or number. IKDC: International Knee Documentation Committee. and 1.7 $\pm 1.2 \mathrm{~mm}$ postoperatively (Table 2 ).

\section{Three-Dimensional Computed Tomography Analysis}

The mean distance from the femoral tunnel center parallel to the Blumensaat's line was $32.5 \% \pm 1.7 \%$ along line $t$ measured from the posterior condylar surface. The mean distance perpendicular to the Blumensaat's line was $36.7 \% \pm 2.1 \%$ along line $\mathrm{h}$ measured from the Blumensaat's line (Table 3 ).

\section{Discussion}

The finding of this study is that the three-point freehand transtibial technique results in a femoral tunnel positioned more posterior and distal to the reference point used in the traditional tanstibial technique. The tunnel position was confirmed by $3 \mathrm{D}$ CT scan at the anatomic footprint ${ }^{12)}$, resulting in good clinical outcome with no instability. The quadrant method is one of the most commonly used techniques for assessing the femoral tunnel position in ACL reconstruction. Using this method in doublebundle ACL reconstruction, Colombet et al. ${ }^{14)}$ reported that the mean distance from proximal condylar surface (line $t$, parallel to Blumensaat's line) was $26.4 \%$ of AM bundle and $32.3 \%$ of posterolateral (PL) bundle (center, 29.35\%), and the mean distance from the notch roof (line h, perpendicular to Blumensaat's line) was $25.3 \%$ of AM and $47.6 \%$ of PL (center, $36.45 \%$ ). Zantop et al. ${ }^{15)}$, Forsythe et al. ${ }^{16)}$, and Tsuda et al. ${ }^{17)}$ also reported similar results (Table 3). Our results were within the range of those studies. Many surgeons adopted the transtibial technique due to the ease of the procedure, reduction in surgical time, rigid fixation and high successful rate ${ }^{18)}$. However, a few studies reported on the difficulty of anatomical femoral tunnel placement at the ACL native footprint with use of the transtibial technique ${ }^{3)}$. The limitations of the offset hook of a femoral guide coupled with the constraints of a tibial tunnel have resulted in a vertical and nonanatomical

Table 3. Comparison of Studies on Femoral and Tibial Positions of Anteromedial (AM) and Posterolateral (PL) Bundles

\begin{tabular}{|c|c|c|c|c|c|c|}
\hline \multirow{3}{*}{ Study } & \multicolumn{6}{|c|}{ Measurements of femoral tunnels } \\
\hline & \multicolumn{3}{|c|}{ Parallel to Blumensaat's line (t) (\%) } & \multicolumn{3}{|c|}{ Perpendecular to Blumensaat's line (h) (\%) } \\
\hline & $\mathrm{AM}$ & PL & Center of ACL & $\mathrm{AM}$ & PL & Center of ACL \\
\hline Colombet et al. ${ }^{14)}$ & $26.4 \pm 2.6$ & $32.3 \pm 3.9$ & 29.35 & $25.3 \pm 4.2$ & $47.6 \pm 6.5$ & 36.45 \\
\hline Fosythe et al. ${ }^{15)}$ & $21.7 \pm 2.5$ & $35.1 \pm 3.5$ & 28.40 & $33.2 \pm 5.6$ & $55.3 \pm 5.3$ & 44.25 \\
\hline Zantop et al. ${ }^{16)}$ & 18.5 & 29.3 & 23.90 & 22.3 & 53.6 & 37.95 \\
\hline Tsuda et al. ${ }^{17)}$ & $25.9 \pm 2$ & $34.8 \pm 2.0$ & 30.35 & $17.8 \pm 2.9$ & $42.1 \pm 3.9$ & 29.95 \\
\hline Current study & & $32.5 \pm 1.7$ & & & $36.7 \pm 2.1$ & \\
\hline
\end{tabular}

Values are presented as mean \pm standard deviation or number.

ACL: anterior cruciate ligament. 
femoral tunnel. Clinical and biomechanical studies demonstrated that a vertically placed graft may restore anterior tibial translation but show less effective resistance to rotatory load compared to lower and more horizontally oriented reconstructions ${ }^{19)}$. Recent biomechanical studies have suggested that a femoral tunnel placed centrally within the native ACL and posteriorly on the lateral wall of intercondylar notch by the trans-AM portal technique can restore both anterior and rotational instability of the knee leading to superior clinical outcomes than traditionally oriented femoral tunnels ${ }^{4}$. Despite the anatomical positioning of the femoral tunnel in the trans-AM portal technique, difficulty of the technique, risk of peroneal nerve injury, risk of short femoral tunnel length and complications associated with hyperflexion of the knee during surgery have been reported ${ }^{8)}$.

The three-point freehand transtibial technique we presented in this study incorporates the advantages of the previously described transtibial technique and the tibial tunnel-independent technique while avoiding the disadvantages and perils associated with each technique. The three-point freehand transtibial technique allows 1) anatomical femoral tunnel placement based on arthroscopic confirmation of the footprint of native ACL, 2) placement of a more oblique femoral tunnel with larger contact surface with the tendon than the previously described TT technique, 3) prevention of short femoral tunnel length, 4) femoral fixation with RigidFix system (DePuy Mitek Inc.) that is much easier and simpler to use and more rigid than other fixation systems such as EndoButton (Smith \& Nephew Inc., Andover, MA, USA), 5) no need for extra time-consuming procedure, 6) more cosmetically pleasing results than the outside-in technique and 7) better visualization than the trans-AM portal technique.

The limitations of this study include the relatively small number of patients, no comparison with the traditional transtibial technique and some limitations inherent to the freehand technique. First, the widening of tibial aperture would happen during femoral tunnel drilling due to the oblique direction. The analysis of tibial tunnel placement and aperture widening was not involved in this study. So, further studies are necessary to address the concerns of tibial tunnel placement and aperture widening. Second, femoral tunnel position is still constrained greatly by the tibial tunnel. So, the position of tibial tunnel is important. Finally, there was a lack of full considerations for the length of the femoral tunnel and the characteristics of the tibial tunnel; however, there was no difficulty in creating a femoral tunnel of $30 \mathrm{~mm}$ in length in all cases and inserting a bioscrew of $25 \mathrm{~mm}$ in length through the tibial tunnel in all cases. We believe studies should be performed to overcome the limitations of our technique and the superiority of the modified transtibial technique over traditional techniques needs to be investigated in further research involving essential clinical evaluation.

\section{Conclusions}

In conclusion, the three-point freehand transtibial technique can be a simple and effective technique that allows for anatomical SB ACL reconstruction.

\section{Conflict of Interest}

No potential conflict of interest relevant to this article was reported.

\section{References}

1. Harner CD, Honkamp NJ, Ranawat AS. Anteromedial portal technique for creating the anterior cruciate ligament femoral tunnel. Arthroscopy. 2008;24:113-5.

2. Piasecki DP, Bach BR Jr, Espinoza Orias AA, Verma NN. Anterior cruciate ligament reconstruction: can anatomic femoral placement be achieved with a transtibial technique? Am J Sports Med. 2011;39:1306-15.

3. Heming JF, Rand J, Steiner ME. Anatomical limitations of transtibial drilling in anterior cruciate ligament reconstruction. Am J Sports Med. 2007;35:1708-15.

4. Bedi A, Musahl V, Steuber V, Kendoff D, Choi D, Allen AA, Pearle AD, Altchek DW. Transtibial versus anteromedial portal reaming in anterior cruciate ligament reconstruction: an anatomic and biomechanical evaluation of surgical technique. Arthroscopy. 2011;27:380-90.

5. Lubowitz JH. Anteromedial portal technique for the anterior cruciate ligament femoral socket: pitfalls and solutions. Arthroscopy. 2009;25:95-101.

6. Gavriilidis I, Motsis EK, Pakos EE, Georgoulis AD, Mitsionis G, Xenakis TA. Transtibial versus anteromedial portal of the femoral tunnel in ACL reconstruction: a cadaveric study. Knee. 2008;15:364-7.

7. Silva A, Sampaio R, Pinto E. ACL reconstruction: comparison between transtibial and anteromedial portal techniques. Knee Surg Sports Traumatol Arthrosc. 2012;20:896-903.

8. Lubowitz JH, Konicek J. Anterior cruciate ligament femoral tunnel length: cadaveric analysis comparing anteromedial portal versus outside-in technique. Arthroscopy. 2010;26:135762. 
9. Nakamura M, Deie M, Shibuya H, Nakamae A, Adachi N, Aoyama H, Ochi M. Potential risks of femoral tunnel drilling through the far anteromedial portal: a cadaveric study. Arthroscopy. 2009;25:481-7.

10. Panni AS, Milano G, Tartarone M, Demontis A, Fabbriciani C. Clinical and radiographic results of ACL reconstruction: a 5- to 7-year follow-up study of outside-in versus inside-out reconstruction techniques. Knee Surg Sports Traumatol Arthrosc. 2001;9:77-85.

11. Rue JP, Ghodadra N, Bach BR Jr. Femoral tunnel placement in single-bundle anterior cruciate ligament reconstruction: a cadaveric study relating transtibial lateralized femoral tunnel position to the anteromedial and posterolateral bundle femoral origins of the anterior cruciate ligament. Am J Sports Med. 2008;36:73-9.

12. Piefer JW, Pflugner TR, Hwang MD, Lubowitz JH. Anterior cruciate ligament femoral footprint anatomy: systematic review of the 21st century literature. Arthroscopy. 2012;28:87281.

13. Bernard M, Hertel P, Hornung H, Cierpinski T. Femoral insertion of the ACL: radiographic quadrant method. Am J Knee Surg. 1997;10:14-21.

14. Colombet P, Robinson J, Christel P, Franceschi JP, Djian P, Bellier G, Sbihi A. Morphology of anterior cruciate ligament attachments for anatomic reconstruction: a cadaveric dissection and radiographic study. Arthroscopy. 2006;22:984-92.

15. Zantop T, Wellmann M, Fu FH, Petersen W. Tunnel positioning of anteromedial and posterolateral bundles in anatomic anterior cruciate ligament reconstruction: anatomic and radiographic findings. Am J Sports Med. 2008;36:65-72.

16. Forsythe B, Kopf S, Wong AK, Martins CA, Anderst W, Tashman S, Fu FH. The location of femoral and tibial tunnels in anatomic double-bundle anterior cruciate ligament reconstruction analyzed by three-dimensional computed tomography models. J Bone Joint Surg Am. 2010;92:1418-26.

17. Tsuda E, Ishibashi Y, Fukuda A, Yamamoto Y, Tsukada H, Ono S. Tunnel position and relationship to postoperative knee laxity after double-bundle anterior cruciate ligament reconstruction with a transtibial technique. Am J Sports Med. 2010;38:698-706.

18. Ahn JH, Park JS, Lee YS, Cho YJ. Femoral bioabsorbable cross-pin fixation in anterior cruciate ligament reconstruction. Arthroscopy. 2007;23:1093-9.

19. Lee MC, Seong SC, Lee S, Chang CB, Park YK, Jo H, Kim $\mathrm{CH}$. Vertical femoral tunnel placement results in rotational knee laxity after anterior cruciate ligament reconstruction. Arthroscopy. 2007;23:771-8. 